\title{
Geometrical error calibration in reflective surface testing based on reverse Hartmann test
}

Zhidong Gong, Daodang Wang, Ping Xu, Chao Wang, Rongguang Liang, et al.

Zhidong Gong, Daodang Wang, Ping Xu, Chao Wang, Rongguang Liang, Ming Kong, Jun Zhao, Linhai Mo, Shuhui Mo, "Geometrical error calibration in reflective surface testing based on reverse Hartmann test," Proc. SPIE 10373, Applied Optical Metrology II, 103730K (23 August 2017); doi: $10.1117 / 12.2268372$

SPIE Event: SPIE Optical Engineering + Applications, 2017, San Diego, California, United States 


\title{
Geometrical error calibration in reflective surface testing based on reverse Hartmann test
}

\author{
Zhidong Gong ${ }^{\mathrm{a}}$, Daodang Wang ${ }^{*}$, Ping Xu ${ }^{\mathrm{a}}$, Chao Wang ${ }^{\mathrm{a}}$, Rongguang Liang ${ }^{\dagger \mathrm{b}}$, Ming Kong ${ }^{\mathrm{a}}$, Jun \\ Zhao ${ }^{\mathrm{a}}$, Linhai $\mathrm{Mo}^{\mathrm{c}}$, Shuhui $\mathrm{Mo}^{\mathrm{c}}$ \\ ${ }^{a}$ College of Metrology and Measurement Engineering, China Jiliang University, Hangzhou 310018, \\ China; ${ }^{b}$ College of Optical Sciences, University of Arizona, Tucson, Arizona 85721, USA; ${ }^{\mathrm{c}}$ Volkslift \\ (China) Company Limited, Huzhou 313009, China
}

\begin{abstract}
In the fringe-illumination deflectometry based on reverse-Hartmann-test configuration, ray tracing of the modeled testing system is performed to reconstruct the test surface error. Careful calibration of system geometry is required to achieve high testing accuracy. To realize the high-precision surface testing with reverse Hartmann test, a computer-aided geometrical error calibration method is proposed. The aberrations corresponding to various geometrical errors are studied. With the aberration weights for various geometrical errors, the computer-aided optimization of system geometry with iterative ray tracing is carried out to calibration the geometrical error, and the accuracy in the order of subnanometer is achieved.
\end{abstract}

Keywords: surface testing; deflectometry; geometrical error calibration; computer-aided optimization

\section{INTRODUCTION}

As an emerging surface type, freeform optical surface has been successfully applied in many applications, such as headmounted displays [1], microlens arrays [2] and bionic compound eye [3], etc. The major advantage of freeform surface is that it makes optical system more compact and more accurate. Compared with traditional rotational symmetric surfaces like spheres and aspheres, freeform surface has higher dynamic range, and it places ultrahigh requirement on the accuracy of measurement tools. Interferometry has served as an accurate and noncontact optical metrology in the measurement of optical surfaces. However, its dynamic range is too small to meet the requirement of most freeform surface to be measured. Besides, complex and expensive compensation optics is generally required in the interferometric testing [4-6].

As a slope measurement method, likes the Ronchi test and Hartmann test, the deflectometry provides a feasible way for freeform surface testing with high dynamic range [7, 8]. By measuring the deflection of reflected beams, the surface slope (derivative of surface sag) can be calculated to reconstruct the test surface. A software configurable optical test system (SCOTS), which is based on fringe reflection/deflectometry and reverse Hartmann test, has been successfully implemented in the testing of large astronomy telescope mirrors and precision X-ray mirrors at the University of Arizona. The deflectometry provides a contact-free, high dynamic range, full field metrology method with simple system setup and alignment [7, 9-14]. However, the achievable testing precision of deflectometry is mainly determined by calibration process. Various approaches have been proposed to achieve accurate calibration of system geometry. Those approaches depend on the high-precision 3D measuring instrument. The calibration process is quite laborious, complicate and time-consuming $[6,15,16]$. In order to achieve high testing accuracy and loose the requirement on the calibration of system geometry, the computer-aided reverse optimization with iterative ray tracing has been proposed. By taking the surface error as the global minimum of the departure from its ideal state, the additive systematic error could be eliminated [7]. However, small residual error introduced by system geometry miscalibration can still be observed in testing results.

\footnotetext{
*wangdaodang@sina.com; phone 86-571-86914563

† rliang@optics.arizona.edu; phone 1-520-621-4995
} 
In this paper, a system geometry calibration method based on Zernike ratio is proposed to lower the requirement on the precision of mechanical device and achieve high measurement precision. The aberrations corresponding to various calibration errors of each component in testing system are discussed in the measurement of freeform surface with the illumination screen and a CCD camera. The rest of this paper is organized as follows: Section 2 presents the principle of reflective surface testing method based on reverse Hartmann test, including the system configuration and basic theory of system geometric calibration based on Zernike ratio. Section 3 shows the numerical analysis and simulation results about the freeform surface testing, and Section 4 draws some concluding remarks.

\section{PRINCIPLE}

\subsection{System layout of reverse Hartmann test}

Hartmann test is a traditional slope measurement method. Figure 1(a) shows the traditional Hartmann test configuration. According to Fig. 1(a), the point light source, which is placed near the curvature center of test mirror, sends the lights through a plate with a number of holes (Hartmann screen) and then the whole test mirror is illuminated. Due to the constraint of the Hartmann screen, the lights passing through the given holes are reflected and then received by the detector. When certain pixels on the detector are lit up, the incident rays and their reflected rays could be defined uniquely. Due to fact that the local surface slopes can be measured by triangulation, the surface image can be obtained from the integration of the slopes $[7,12]$.
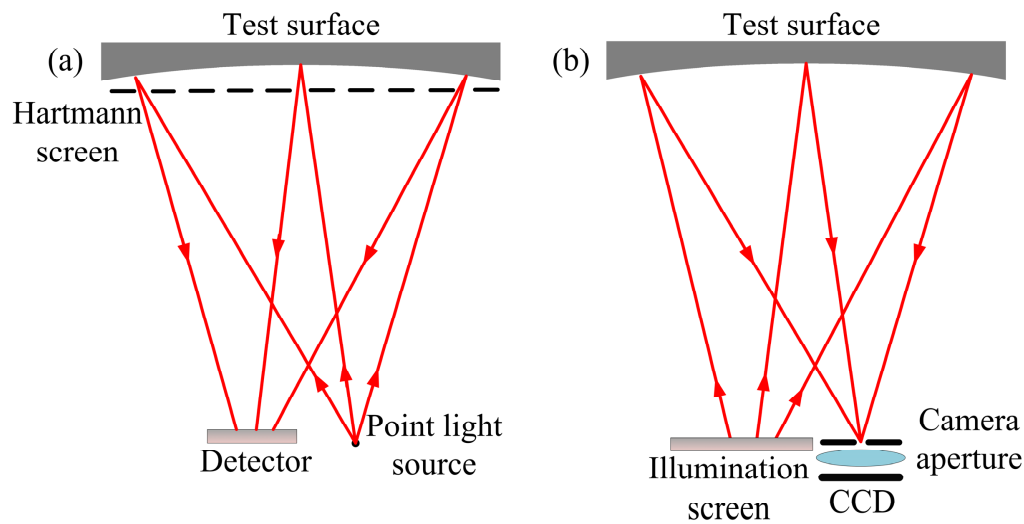

Fig. 1. System layout. (a) Hartmann test, (b) Reverse Hartmann test.

The basic geometry of testing system based on reverse Hartmann test is similar to Hartmann test, as is shown in Fig. 1(b). According to Fig. 1(b), the CCD is employed as the point source in Hartmann test to detect the light reflected from test surface. The detector is replaced by an LCD screen. When an illuminating screen pixel is lit up, the image on the CCD will show a bright region corresponding to a certain reflection region on test surface. The direction of the incident and reflected light is determined by the coordinate of these three points uniquely. To realize the quick obtainment of surface normal map, the sinusoidal fringes illumination and phase shifting method is applied [17, 18].

In the testing of a polished optical surface, the key point is to obtain the surface departure from its ideal shape. The virtual null test can be realized by setting up a ray tracing model, in which the test optics is set to its ideal shape. By ray tracing the testing system with ideal surface, an ideal spot distribution $\left(x_{\text {model }}, y_{\text {model }}\right)$ on the image plane can be obtained. In the experiment, the actual spot distribution $\left(x_{\text {test }}, y_{\text {test }}\right)$ is calculated from the sinusoidal-fringe phase-shifting method. According to the transverse ray aberration model, the system wavefront aberration can be approximately equal to the transverse ray aberration [10]. The slope differences $\left(\Delta w_{x}, \Delta w_{y}\right)$ can be obtained by dividing the spot coordinate differences $\left(\Delta x_{s p o t}, \Delta y_{s p o t}\right)$ with the mirror-to-screen distance $d_{m 2 s}$, 


$$
\left\{\begin{array}{l}
\Delta w_{x}=\frac{\partial W(x, y)}{\partial x}=\frac{x_{\text {test }}-x_{\text {model }}}{2 d_{m 2 s}}=\frac{\Delta x_{\text {spot }}}{2 d_{m 2 s}} \\
\Delta w_{y}=\frac{\partial W(x, y)}{\partial y}=\frac{y_{\text {test }}-y_{\text {model }}}{2 d_{m 2 s}}=\frac{\Delta y_{\text {spot }}}{2 d_{m 2 s}}
\end{array}\right.
$$

where $(x, y)$ are the exit pupil coordinate of the system, $W(x, y)$ refers to the wavefront aberration. With the surface integration, the surface error map can be reconstructed from the slope differences $\left(\Delta w_{x}, \Delta w_{y}\right)$.

\subsection{Geometrical aberrations in reverse Hartmann test}

In the configuration of reverse Hartmann test, which is an off-axis system setup, both the illumination screen and camera are laterally displaced from the optical axis of test surface. The calibration error of the geometrical relations among various components in the system, including the tilt angle deviations, lateral and longitudinal displacements, could introduce evident residual error in the testing result, even though the measurement accuracy of calibration device can reach the order of microns. Taking the lateral displacement and tilt around $x$ axis as examples, they would result in the off-axis aberrations including astigmatism, spherical and coma, respectively. As a classical description of wavefront, the Zernike polynomials, in which the system wavefront data $W$ can be expressed with a series of orthonormal polynomials $Z_{i}(\rho, \theta)$ with coefficients $C_{i}$, is applied for the off-axis aberrations analysis [19],

$$
W=\sum_{i=1}^{N}\left[C_{i} Z_{i}(\rho, \theta)\right]
$$

where $N$ is the total term number of Zernike polynomials, $(\rho, \theta)$ are the polar coordinates on the test surface.

For the convex spherical surface with an aperture diameter of $50.8 \mathrm{~mm}$ and curvature radius of $250 \mathrm{~mm}$, Table 1 shows that the correspondence between Zernike coefficients and the off-axis aberrations. In traditional methods, the major systematic error introduced by system geometrical error can be removed from testing result, simply by setting the corresponding low-order Zernike coefficients to zero [20]. However, some residual high-order aberrations can be observed in the testing result according to Table 1.

Table 1. Correspondence between Zernike coefficients and the off-axis aberrations.

\begin{tabular}{|l|l|l|}
\hline Zernike polynomials & \multicolumn{1}{|c|}{ Aberrations } & Zernike coefficients $(\boldsymbol{\mu m})$ \\
\hline Z4 & Astig $x$ & -1.9354 \\
\hline Z5 & Astig y & 0.8275 \\
\hline Z6 & Coma $x$ & -0.6286 \\
\hline Z7 & Coma y & 0.9543 \\
\hline Z8 & Primary Spherical & -0.1807 \\
\hline Z9 & Trefoil $x$ & -0.6577 \\
\hline Z10 & Trefoil y & -1.4360 \\
\hline Z11 & Secondary Astigmatism x & -0.9443 \\
\hline Z12 & Secondary Astigmatism y & -0.7888 \\
\hline Z13 & Secondary Coma $x$ & 0.1098 \\
\hline
\end{tabular}

Figure 2 shows the calibration error of the lateral displacement along $x$ axis and tilt deviation around $x$ axis, which would introduce significant residual aberrations. According to Fig. 2, the maximum variation of Zernike coefficient corresponding to $0.1 \mathrm{~mm}$ lateral displacement calibration error reaches $0.1314 \mu \mathrm{m}$, and that for 0.1 degree tilt calibration 
error is about $0.8971 \mu \mathrm{m}$. Thus, it is necessary to study the aberrations corresponding to various calibration errors of each component in testing system.

In the previous work, a computer-aided system geometry calibration method based on the ray tracing of the testing system was proposed, and the surface error was taken as the global minimum of the departure from its ideal shape [7]. However, the diversity and mutual influence of error factors would affect the optimization result, and further calibration is required to minimize the residual systematic error.
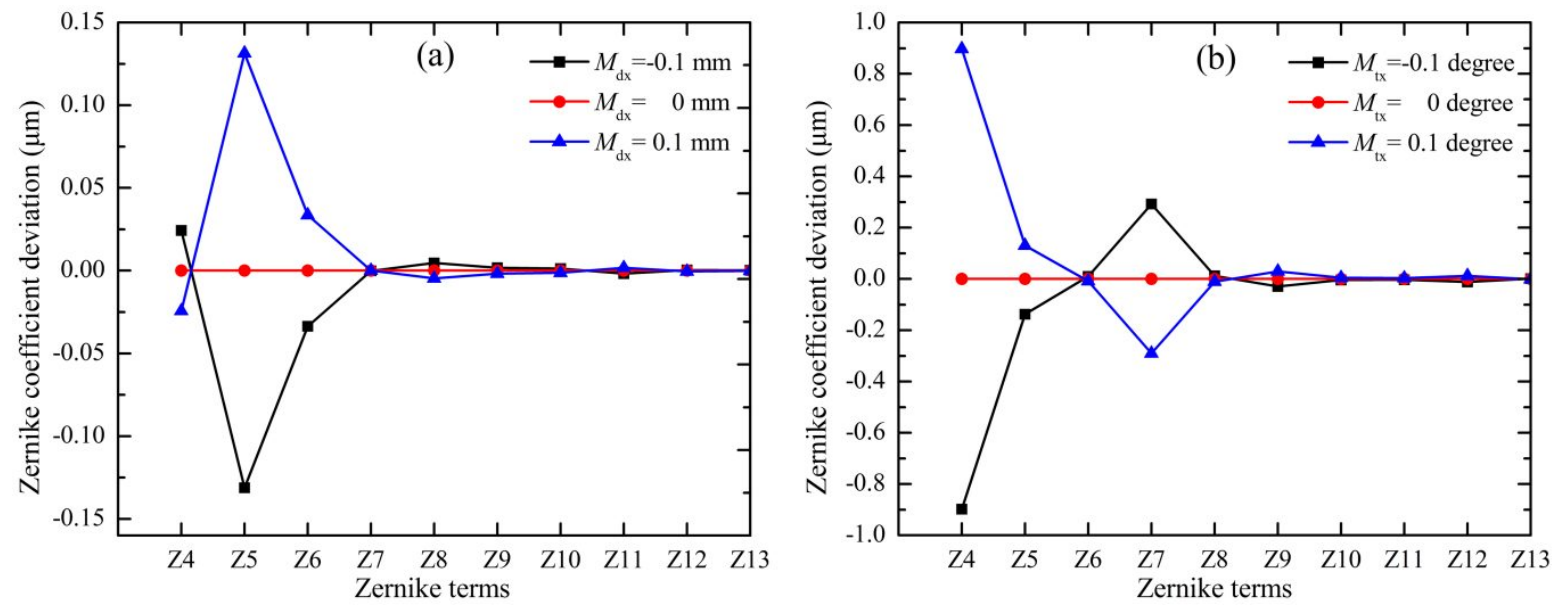

Fig. 2. Zernike coefficients caused by geometrical error. (a) Lateral displacement along $x$ axis and (b) tilt angle deviation around $x$ axis of the convex surface with the $25.4 \mathrm{~mm}$ semi-diameter and $250 \mathrm{~mm}$ curvature radius.

\subsection{System geometric calibration based on Zernike ratio}

The calibration of system geometrical error can be realized by ray tracing of testing system, as well as to achieve the virtual null testing of surface error. Accordingly, the initial measurement result $W_{t e s t}^{(0)}$, which is measured in the testing system model, can be expressed with a series of orthonormal polynomials $Z_{i}(\rho, \theta)$ with coefficients $C_{i, \text { test }}^{(0)}$,

$$
W_{\text {test }}^{(0)}=W_{\text {geo }}+W_{\text {surf }}=\sum_{i=1}^{N}\left[C_{i, \text { test }}^{(0)} Z_{i}(\rho, \theta)\right]
$$

where $W_{\text {geo }}$ and $W_{\text {surf }}$ are the systematic error introduced by system geometry and test surface error, respectively.

Due to the fact that the geometrical error is introduced by various system parameters, including the tilts in $x, y$ and $z$ directions, decenter in $x$ and $y$ directions, axial displacement of test surface relative to camera aperture, and the same errors of illumination screen relative to test surface. It is difficult to separate the geometric error from the measured data. To obtain the aberration weights $r_{i}^{(j)}$ for various geometrical errors, an addition deviation of single system parameter from its original value was added to the ray-tracing system model, and we have the measurement result

$$
W_{\text {test }}^{(j)}=W_{\text {geo }}+W_{\text {surf }}+W_{\Delta}^{(j)}=\sum_{i=1}^{N}\left[C_{i, t e s t}^{(j)} Z_{i}(\rho, \theta)\right]
$$

where $j$ refers to the $j_{t h}$ single component among system parameters, $W_{\Delta}^{(j)}$ is the wavefront aberration introduced by the increment of the $j_{t h}$ single component. Thus, the wavefront aberration $W_{\Delta}^{(j)}$ can be fitted to Zernike polynomials as

$$
W_{\Delta}^{(j)}=W_{\text {test }}^{(j)}-W_{\text {test }}^{(0)}=\sum_{i=1}^{N}\left\{\left[C_{i, \text { test }}^{(j)}-C_{i, \text { test }}^{(0)}\right] Z_{i}(\rho, \theta)\right\}=\sum_{i=1}^{N}\left[\Delta C_{i}^{(j)} Z_{i}(\rho, \theta)\right],
$$

where $\Delta C_{i}^{(j)}$ is Zernike coefficients corresponding to the wavefront aberration $W_{\Delta}^{(j)}$. 
As is shown in Fig. 2, obvious function correlativity exists between the coefficients of Zernike polynomials and the variation of each single component among system parameters. Under the conditions of the freeform surface and small working distance, the obvious systematic error due to each $j_{t h}$ single system geometry calibration error $\varepsilon_{j}$ could be estimated according to the functional relationship $\psi_{i, j}\left(\varepsilon_{j}\right)$ between them, where the subscript $i$ indicates the $i_{t h}$ Zernike coefficient of systematic error. Based on linear approximations, the aberration weights $r_{i}^{(j)}$ for various geometrical errors can be expressed as

$$
r_{i}^{(j)}=C_{i}^{(j)} / C_{3}^{(j)}=\Delta C_{i}^{(j)} / \Delta C_{3}^{(j)},
$$

where $C_{i}^{(j)}$ is the $i_{t h}$ coefficient of Zernike polynomials with the influence of the $j_{t h}$ single component. The Zernike coefficient $\Delta C_{i}^{(j)}$ can be obtained with the differential method in Eq. (5), and we have the aberration weights $r_{i}^{(j)}$

$$
r_{i}^{(j)}=\left[C_{i, \text { test }}^{(0)}-C_{i, \text { surf }}+\sum_{k \neq j}^{m} \psi_{i, k}\left(\varepsilon_{k}\right)\right] /\left[C_{i, \text { test }}^{(0)}-C_{3, \text { surf }}+\sum_{k \neq j}^{m} \psi_{3, k}\left(\varepsilon_{k}\right)\right],
$$

where $C_{i, \text { surf }}$ are the coefficients for the orthogonal polynomials fitting of modeled wavefront aberration introduced by surface error, $m$ is the total number of the components among system parameters.

With the initial measurement result $W_{\text {test }}^{(0)}$ and the known functional relationships $\psi_{i, k}\left(\varepsilon_{k}\right)$, the measurement result $W_{\text {test }}^{(k)}$, which is influenced by all the components, can be obtained by adjusting the model parameters. According to the Eq. (7), the restrictive condition can be applied to obtain the coefficients $\left\{C_{i, \text { sur }}\right\}$ of Zernike polynomials,

$$
\sum_{k \neq j}^{m}\left[\psi_{i, k}\left(\varepsilon_{k}\right)-r_{i, j_{0}} \psi_{3, k}\left(\varepsilon_{k}\right)\right]=r_{i}^{(j)} \cdot\left[C_{i, \text { test }}^{(0)}-C_{3, \text { surf }}\right]-\left[C_{i, \text { test }}^{(0)}-C_{i, \text { surf }}\right],
$$

where $\left[C_{i, t e s t}^{(0)}-C_{i, \text { surf }}\right]$ is the objective and it can be obtained from the overdetermined linear function shown in Eq. (8) with the least square method.

Due to the fact that the original coefficients $C_{i, \text { test }}^{(0)}$ is known, the modeled coefficient $C_{i, \text { surf }}$ can be calculated to describe the surface error. Under the restrictive condition in Eq. (7), the surface error can be obtained when the geometrical error is the global minimum of the departure from its ideal shape. In the reverse optimization method, the geometrical parameters of test system can be optimized according to the objective function,

$$
F\left(\varepsilon_{j}\right)=\min \left[\left(W_{\text {surf }}\right)^{2}+c\right]=\min \left[\sum_{j=1}^{N}\left(C_{i, \text { surf }}\right)^{2}+c\right],
$$

where the parameter $c$ is an additional constraint to restrict the solution space. With the optimal solution $\varepsilon_{j}^{*}$ after optimization, the test surface error $W_{\text {surf }}$ can be estimated as

$$
W_{\text {surf }}=W_{\text {test }}-W_{\text {geo }}\left(\varepsilon_{j}^{*}\right) .
$$

Figure 3 exhibits the procedure for the geometrical error calibration method based on Zernike ratio. When the experimental system is set up, the pre-calibrated system geometrical parameters, which is measured by three-dimensional positioning equipment like CMM, can be employed to build a testing system model in the ray-tracing software. Approximately, the wavefront aberration $W_{\text {test }}^{(0)}$, which is measured in the reverse Hartmann test, can be taken as the summation of simulated systematic error $W_{\text {geo }}$ and test surface error $W_{\text {surf }}$. Subsequently, the constant Zernike ratio $r_{i}^{(j)}$ and the functions $\psi_{i, j}\left(\varepsilon_{j}\right)$ are built by constantly adjusting the geometric parameters in the ray-tracing software according to Eq. (6). In the optimization procedure, the pre-calibrated system parameters are taken as the original value, and the geometrical deviation $\varepsilon_{j}$ is employed to update the wavefront in the model with the functions $\psi_{i, j}\left(\varepsilon_{j}\right)$. When 
the objective function $F\left(\varepsilon_{j}\right)$ reaches the threshold $\delta$, the optimal geometrical deviation $\varepsilon_{j}^{*}$ and the corresponding test surface error $W_{\text {surf }}$ can be obtained.

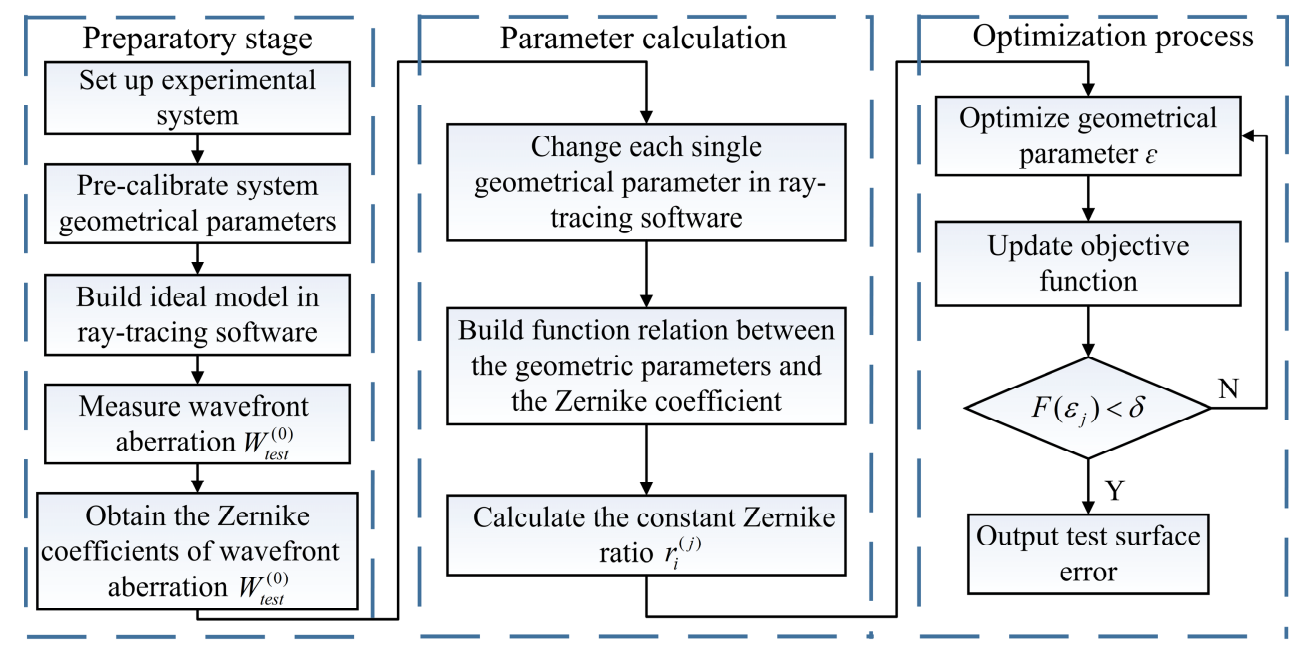

Fig. 3. Procedure for system geometry calibration.

\section{NUMERICAL SIMULATION}

To validate the feasibility and accuracy of the proposed method for the geometrical error calibration in reflective surface testing based on reverse Hartmann test, the ray-tracing computer simulation is carried out, in which the wavefront aberration is characterized with 37-term Zernike coefficients. A test freeform surface is employed to build a reverse Hartmann test system with the configuration shown in Fig. 1(b) in the ray-tracing software (ZEMAX). The actual test surface error is shown in Fig. 4(a), whose peak-to-valley (PV) and root-mean-square (RMS) values are $18.5591 \mu \mathrm{m}$ and $3.4657 \mu \mathrm{m}$, respectively. Figure 4(b) shows the whole surface map of test freeform surface, whose aperture diameter is $50.8 \mathrm{~mm}$ and PV value is $1.2832 \mathrm{~mm}$. The best fit sphere vertex offset of test surface is $-6.4021 \mu \mathrm{m}$, and the distance $d_{m 2 s}$ between the mirror and the screen is $151.9310 \mathrm{~mm}$.

To analyze the effect of system geometry calibration error on the testing result, the systematic error introduced by system geometry calibration error is studied in detail. Figure 5 shows the wavefront aberrations corresponding to various calibration errors of the lateral displacement $D_{m x}$ along $x$ axis and tilt $T_{m x}$ about $x$ axis for the test surface. According to Fig. 5, the systematic error grows linearly with the lateral displacement error along $\mathrm{x}$ axis and tilt error from its original value, respectively. In the freeform surface testing, the RMS value of residual error corresponding to $20 \mu \mathrm{m}$ lateral displacement calibration error is about $0.0069 \mu \mathrm{m}$, and that for 0.01 degree tilt calibration error can reach $0.0239 \mu \mathrm{m}$.

In the simulation, an additional deviation of the lateral displacement along $x$ axis $D_{m x}=-0.06 \mathrm{~mm}$ and tilt about $x$ axis $T_{m x}=-0.103^{\circ}$ from its original value are added to the test surface in the ray tracing model, in which the test surface is set as an ideal shape. According to the testing method introduced in Section 2, the virtual "null" test of ideal surface can be carried out to obtain the initial measurement result $W_{\text {test }}^{(0)}$ in Eq. (3), as is shown in Fig. 4(c). Figure 4(d) shows the residual error of the initial measurement result $W_{\text {test }}^{(0)}$ (Fig. 4(c)) with respect to the actual surface error (Fig. 4(a)), in which a significant residual error with the PV value $6.0458 \mu \mathrm{m}$ and RMS value $0.9848 \mu \mathrm{m}$ can be observed. 

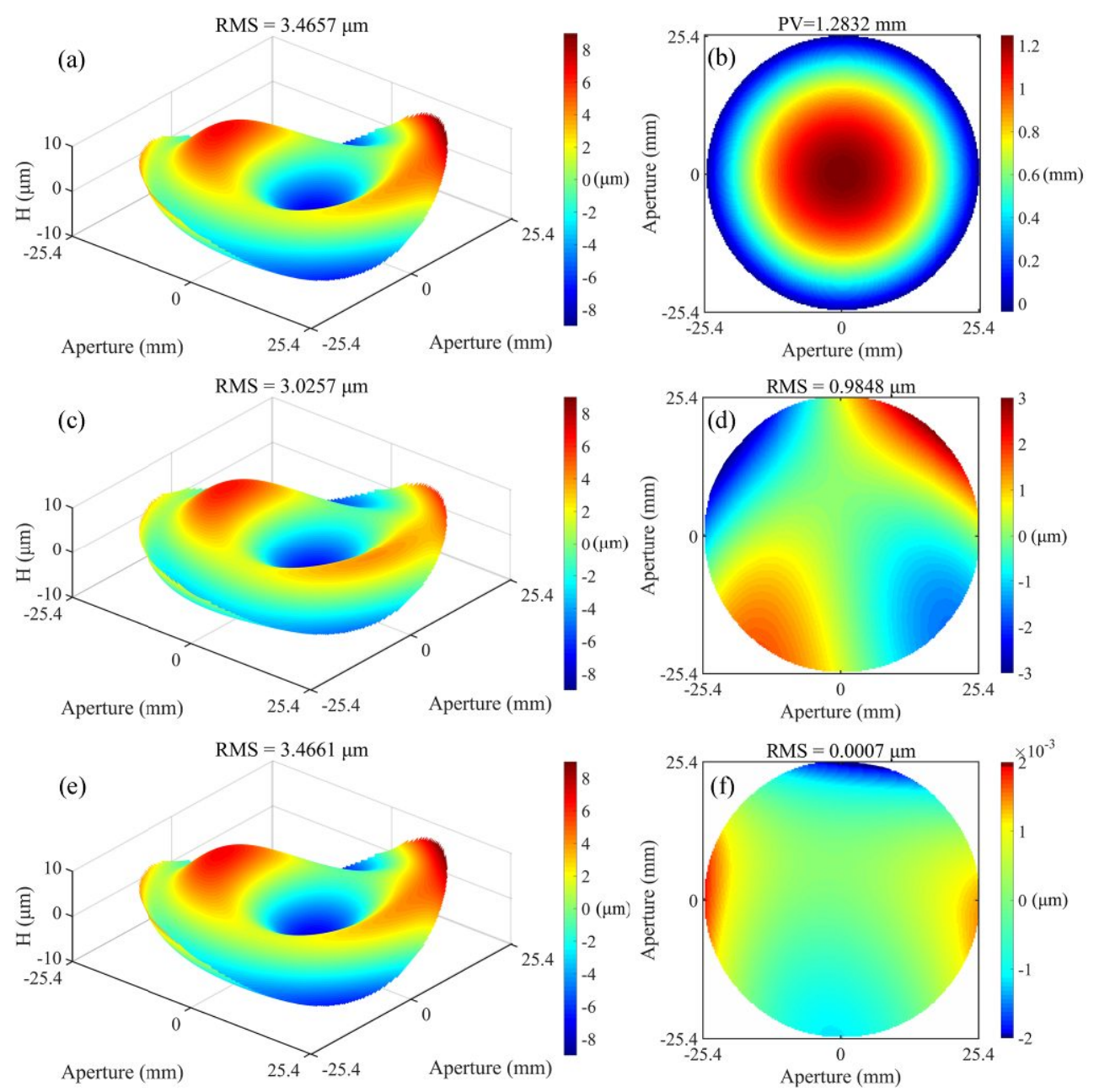

Fig. 4. Surface testing results in the simulation. (a) Actual surface error of test freeform surface, (b) test surface, (c) test surface error and (d) the corresponding residual error with existence of system geometry measurement error, (e) test surface error and (f) the corresponding residual error after system geometry calibration.
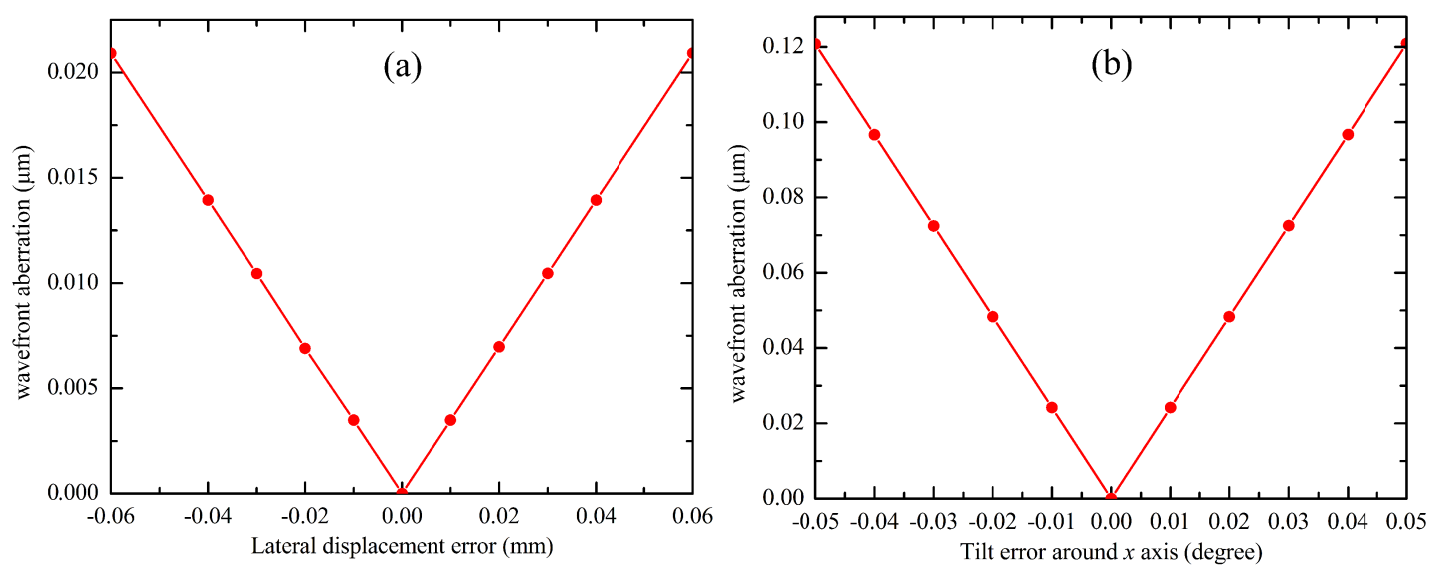

Fig. 5. Systematic error introduced by system geometry calibration error in the simulation. Wavefront aberrations corresponding to (a) lateral displacement error along $x$ axis and (b) tilt error around $x$ axis. 
Then the system geometry calibration process introduced in Fig. 3 is performed to remove the system geometry calibration error. After the reverse optimization of the system geometry, the optimization result about test surface error is shown in Fig. 4(e), and Fig. 4(f) is the corresponding residual error with the PV and RMS values $0.0039 \mu \mathrm{m}$ and 0.0007 $\mu \mathrm{m}$, respectively. The deviation of lateral displacement along $x$ axis $D_{m x}$ and tilt about $x$ axis $T_{m x}$ are $0.6 \mu \mathrm{m}$ and 0.0001 degree, respectively. Table 2 presents the detailed value about the freeform surface testing results.

Table 2. PV and RMS values of surface testing results in the simulation.

\begin{tabular}{|l|l|l|}
\hline \multicolumn{1}{|l|}{} & \multicolumn{1}{|c|}{ PV ( $\boldsymbol{\mu m})$} & \multicolumn{1}{c|}{ RMS $(\boldsymbol{\mu m})$} \\
\hline Actual surface error & 18.5591 & 3.4657 \\
\hline With system geometric error & 13.9202 & 3.0257 \\
\hline Test surface error & 6.0458 & 0.9848 \\
\hline Residual error & 18.5635 & 3.4661 \\
\hline After system geometry calibration & 0.0007 \\
\hline Test surface error & 0.0041 &
\end{tabular}

According to Fig.4 and Table 2, the computer simulation results confirm the accuracy and feasibility of the proposed calibration system. Moreover, it provides a high-precision method to measure freeform surface, whose surface departure is within the dynamic range of deflectometry. Besides, this method provides a feasible way to lower the requirement on the calibration of system geometry, and is of great practicality for the high-precision measurement of freeform surface.

\section{CONCLUSION}

In this paper, the geometric calibration errors in the surface testing based on reverse Hartmann test, including the tilt angle deviations, lateral and longitudinal displacements (both test surface and screen), are studied in the ray tracing model. According to the analysis, system geometric calibration based on Zernike ratio provides a feasible way to improve the measurement accuracy. The numerical simulation has been carried out to demonstrate the feasibility of the proposed optimization method, which enables high-precision testing of freeform surfaces. In addition, system geometric calibration based on Zernike ratio is a feasible way to loose the requirement on the calibration of system geometry.

\section{ACKNOWLEDGEMENTS}

The activities of this work are supported by Zhejiang Provincial Natural Science Foundation of China (LY17E050014), National Natural Science Foundation of China (NSFC) (51375467, 11404312, 51476154, 51404223), Zhejiang Key Discipline of Instrument Science and Technology (JL150508).

\section{REFERENCES}

[1] Yang, T., Zhu, J. and Jin, G., "Compact freeform off-axis three-mirror imaging system based on the integration of primary and tertiary mirrors on one single surface," Chinese Optics Letters 14(6), 060801 (2016).

[2] Zhu, J., Hou, W., Zhang, X. and Jin, G., "Design of a low F-number freeform off-axis three-mirror system with rectangular field-of-view," Journal of Optics 17(1), 015605 (2014).

[3] Pang, K., Fang, F., Song, L., Zhang, Y. and Zhang, H., "Bionic compound eye for 3D motion detection using an optical freeform surface," Journal of the Optical Society of America B 34(5), B28-B35 (2017).

[4] Otaki, K., Ota, K., Nishiyama, K., Yamamoto, T., Fukuda, Y. and Okazaki, S., "Development of the point diffraction interferometer for extreme ultraviolet lithography: Design, fabrication, and evaluation," Journal of Vacuum Science and Technology B 20(6), 2449-2458 (2002).

[5] Wang, D., Yang, Y., Chen, C. and Zhuo, Y., "Point diffraction interferometer with adjustable fringe contrast for testing spherical surfaces," Applied Optics 50(16), 2342-2348 (2011). 
[6] Wang, D., Yang, Y., Chen, C. and Zhuo, Y., "Calibration of geometrical systematic error in high-precision spherical surface measurement," Optics Communications 284(16-17), 3878-3885 (2011).

[7] Wang, D., Zhang, S., Wu, R., Huang, C., Cheng, H. and Liang, R., "Computer-aided high-accuracy testing of reflective surface with reverse Hartmann test," Optics Express 24(17), 19671-19681 (2016).

[8] Knauer, M., Kaminski, J. and Hausler, G., "Phase measuring deflectometry: a new approach to measure specular free-form surfaces," Proc. SPIE 5457, 366-76 (2004).

[9] Su, P., Parks, R., Wang, L., Angel, R. and Burge, J., "Software configurable optical test system: a computerized reverse Hartmann test," Applied Optics 49(23), 4404-4412 (2010).

[10] Su, P., Wang, Y., Burge, J., Kaznatcheev, K. and Idir, M., "Non-null full field X-ray mirror metrology using SCOTS: a reflection deflectometry approach," Optics Express 20(11), 12393-12406 (2012).

[11] Huang, R., Su, P., Horne, T., Brusa, G. and Burge, J., "Optical metrology of a large deformable aspherical mirror using software configurable optical test system," Optical Engineering 53(8), 085106 (2014).

[12] Huang, R., Su, P., Burge, J., H. Huang, L. and Idir, M., "High-accuracy aspheric x-ray mirror metrology using Software Configurable Optical Test System/deflectometry," Optical Engineering 54(8), 084103 (2015).

[13] Dominguez, M., Armstrong, J., Su, P., Parks, R. and Burge, J., "SCOTS: a useful tool for specifying and testing optics in slope space," Proc. SPIE 8493, 84931D (2012).

[14] Su, P., Khreishi, M., Su, T., Huang, R., Dominguez, M., Maldonado, A. and Burge, J., "Aspheric and freeform surfaces metrology with software configurable optical test system: a computerized reverse Hartmann test," Optical Engineering 53(3), 031305-031305 (2014).

[15] Wang, D., Yang, Y., Chen, C. and Zhuo, Y., "Misalignment aberrations calibration in testing of high-numericalaperture spherical surfaces," Applied Optics 50(14), 2024-2031 (2011).

[16] Huang, L., Chua, P. and Asundi, A., "Least-squares calibration method for fringe projection profilometry considering camera lens distortion," Applied Optics 49(9), 1539-1548 (2010).

[17] Tomasz, T. and JoÂ, R., "Influence of optical imaging on phase measurements in fringe projection coherent systems," Optical Engineering 41(4), 811-821 (2002).

[18] Andraka, C., Sadlon, S., Myer, B., Trapeznikov, K. and Liebner, C., "Rapid reflective facet characterization using fringe reflection techniques," Journal of Solar Energy Engineering 136(1), 011002 (2013).

[19] Roddier, N., "Atmospheric wavefront simulation using Zernike polynomials," Optical Engineering 29(10), 1174-1180 (1990).

[20] Rayces, J., "Exact relation between wave aberration and ray aberration," Journal of Modern Optics 11(2), 85-88 (1964). 